\title{
THE TJALLING C. KOOPMANS ECONOMETRIC THEORY PRIZE: 1991-1993
}

Econometric Theory is proud to announce joint winning articles for the Tjalling C. Koopmans Econometric Theory Prize over the period 19911993. The prize is jointly supported by the publishers, Cambridge University Press, and Mrs. Truus Koopmans. It is named in honor of Tjalling C. Koopmans, the 1975 Nobel Laureate in Economic Science. The selection of the winning articles was made by the Advisory Board of the journal and all articles published in Econometric Theory (1991-1993) were candidates for the prize, except those that were authored or coauthored by the Editor and members of the Advisory Board. The prize is accompanied by a financial award of $\$ 500$ for each of the winning articles.

The winning articles and citations (written by the Advisory Board) are as follows:

Pentti Saikkonen. (1993) Estimation of cointegration vectors with linear restrictions. Econometric Theory 9(1), 19-35

This paper develops a general method of estimating and testing cointegration vectors, with linear restrictions. In the case of zero restrictions, the cointegration relations are formally similar to the structural equations of a traditional simultaneous equations model, and the paper provides an important link between the literature on statistical inference in simultaneous equations models and the more recent literature on cointegration analysis. The asymptotic distribution of the estimators are shown to be mixed normal, so that Wald tests with asymptotic chi-square distributions under the null hypothesis are obtained in the usual way. Convenient test procedures for checking the validity of overidentifying restrictions are also provided.

Katsuto Tanaka. (1993) An alternative approach to the asymptotic theory of spurious regression, cointegration, and near cointegration. Econometric Theory 9(1), 36-61

This paper uses the Fredholm approach to derive new expressions for the asymptotic sampling distributions of estimators and test statistics in cointegration models. It is shown that, in some cases, these expressions provide a basis for the accurate computation of the limiting distributions. The paper also introduces a definition of near cointegration, for which asymptotic properties are studied. It then devises tests that take cointegration as the null hypothesis and discusses the limiting local power of these tests under the alternative of near cointegration.

Mrs. Truus Koopmans and Cambridge University Press join me in congratulating the authors on their success in receiving this award.

Peter C.B. Phillips

New Haven, CT, July 6, 1996 


\section{THE LEONARD J. SAVAGE AWARD}

Members of the NBER-NSF Seminar on Bayesian Inference in Econometrics and Statistics, the International Society for Bayesian Analysis, and the ASA Section on Bayesian Statistical Science are cosponsoring an annual Leonard J. Savage Award of $\$ 750$ for an outstanding doctoral dissertation in the area of Bayesian Econometrics and Statistics.

To be considered for the 1997 Savage Award, a doctoral dissertation must be submitted by the dissertation supervisor before December 31, 1997, and accompanied by a short letter from the supervisor summarizing the main results of the dissertation. Dissertations completed after January 1, 1977, are eligible to be considered for the 1997 Savage Award. An evaluation committee will be appointed by the board of the Leonard J. Savage Memorial Trust Fund (S.E. Fienberg, S. Geisser, J.B. Kadane, E.E. Leamer, J.W. Pratt, and A Zellner, Chairman) to evaluate dissertations that are submitted for the Savage award.

Dissertations and supporting letters should be sent to Professor Arnold Zellner, Graduate School of Business, University of Chicago, 1101 East 58th Street, Chicago, IL 60637, USA.

The 1996 winners will be announced in the near future. The co-winners of the 1995 Savage Award are Alyson Wilson for her thesis, "Statistical Models for Shapes and Deformations," completed at Duke University under the direction of Valen Johnson, and Christopher Carter for his thesis, "Markov Chain Monte Carlo Methods for State Space Models," completed at the University of New South Wales, Australia, under the direction of Robert Kohn.

The following candidates were accorded Honorable Mention: Ming-Hui Chen, "Monte Carlo Markov Chain Sampling for Evaluating Multivariate Integrals with Applications to Bayesian Computation," completed at Purdue University under the direction of James Berger, and Simon J. Godsill, "Bayesian Enhancement of Speech and Audio Signals in the Presence of Both Impulsive and Background Noise," completed at Cambridge University under the direction of P.J.W. Rayner. 